\title{
Olfactory impairment in first-episode schizophrenia: a case-control study, and sex dimorphism in the relationship between olfactory impairment and psychotic symptoms
}

\author{
Xiacan Chen ${ }^{1+}$, Jiajun $\mathrm{Xu}^{2^{*+}} \mathbb{D}$, Bin $\mathrm{Li}^{2}$, Wanjun $\mathrm{GuO}^{2}$, Jun Zhang ${ }^{2}$ and Junmei $\mathrm{Hu}^{{ }^{*}}$
}

\begin{abstract}
Background: A body of studies has focused on the olfactory impairment among people with schizophrenia. The effect of sex on this relationship has attracted the attention of researchers. These issues have not been studied much in Chinese schizophrenia patients.

Methods: We conducted a case-control study of 110 first-episode antipsychotic medicine naïve schizophrenia patients aged 18-35 years and 110 controls, matched by age and sex. Odour threshold, discrimination and identification were assessed by the "Sniffin' Sticks" test. Psychotic symptoms were assessed by the Positive and Negative Syndrome Scale (PANSS).

Results: The odour threshold, discrimination and identification scores of patients with schizophrenia were significantly lower than those of the healthy control group. The difference in identification score had statistical significance between male and female patients with schizophrenia $(t=-2.45, P<0.05)$. Controlling for confounding factor, in male schizophrenia participants, the negative subscale score was significantly and inversely correlated with the discrimination $(\gamma=-0.37, p<0.008)$, identification $(\gamma=-0.45, p<0.008)$ and TDI $(\gamma=-0.50, p<0.008)$ scores; the general psychopathology subscale score was inversely and significantly correlated with the identification $(\gamma=-0.47, p<0.008)$ and TDI $(\gamma=-0.41, p<0.008)$ scores. For female schizophrenia patients, positive and general psychopathology subscale scores had a significant inverse correlation with the identification score (positive: $\gamma=-0$. 47, $p<0.008$; general psychopathology: $\gamma=-0.42, p<0.008$ ).

Conclusions: Controlling for confounder, negative symptoms were related to impaired odour discrimination and identification in male schizophrenia patients, while positive symptoms were correlated with impaired odour identification in female schizophrenia patients. This sex dimorphism could provide useful information for future studies aiming to finding biomarkers of schizophrenia.
\end{abstract}

Keywords: Schizophrenia, Olfaction, Sex, Negative symptom, Positive symptom

* Correspondence: xujiajun120@126.com; junmeihu@hotmail.com

${ }^{+}$Xiacan Chen and Jiajun Xu contributed equally to this work.

${ }^{2}$ Mental Health Center, West China Hospital, Sichuan University, Sichuan,

China

${ }^{1}$ West China School of Basic Medical Sciences \& Forensic Medicine, Sichuan

University, Sichuan, China

(c) The Author(s). 2018 Open Access This article is distributed under the terms of the Creative Commons Attribution 4.0 International License (http://creativecommons.org/licenses/by/4.0/), which permits unrestricted use, distribution, and reproduction in any medium, provided you give appropriate credit to the original author(s) and the source, provide a link to the Creative Commons license, and indicate if changes were made. The Creative Commons Public Domain Dedication waiver (http://creativecommons.org/publicdomain/zero/1.0/) applies to the data made available in this article, unless otherwise stated. 


\section{Background}

Schizophrenia, mainly defined by its psychotic symptoms, lacks physiological examination to diagnosis [1]. Many studies were conducted with the aim of finding biomarkers of schizophrenia and olfactory function has been one of the directions. Olfaction, an age- and sex-related function, is one of the most direct neural pathways between external environment and the central nervous system. There is overlap of some neuroanatomical structure with the impaired brain region in those with schizophrenia, such as the medial temporal lobe, amygdala and hippocampus [1-3]. Furthermore, the olfactory function examination was more convenient and reliable than a review of the patient symptom state which is subjective and always reported by patients themselves. Lastly, olfactory impairment was found in schizophrenia patients, and has been especially correlated with negative symptoms [4, 5]. Consequently, an understanding olfaction would be useful for the clinical practice of schizophrenia.

Olfaction is a multiple sensory process, and the deficit of it can be divided into peripheral and central abnormalities. Odour detection threshold (sensitivity) is more peripheral, while identification and discrimination are more central [6-8]. Olfactory dysfunction is well documented in schizophrenia patients and odour identification impairment has been suggested as a vulnerability marker of deficit syndrome in some studies [5, 9], while a meta-analysis failed to find evidence for considering olfaction identification as vulnerability marker of schizophrenia [10]; however, the absence of accounting for sex effect in the meta-analysis could profoundly influence the results, for sex difference in olfaction have been documented not only in healthy individuals, but also in schizophrenia patients $[11,12]$. Many studies were conducted with small sample sizes and these multiple olfactory functions (e.g., threshold, identification and discrimination) were barely examined in same subjects, as Moberg noted [13]; in addition, odour threshold and discrimination were reported much less among patients with schizophrenia when comparing with odour identification [4, 7].

Sexual dimorphism is common in neuroscience and schizophrenia studies. The differences of manifestations, social function and brain structure have been addressed between male and female among schizophrenia [14-17]. Sex difference was also presented in olfactory performance among patients with schizophrenia, in that some researchers have reported that male schizophrenia patients show a strong relation between negative symptoms and odour identification, while female patients have failed to present this relation $[12,18]$. However, the relation between psychotic symptoms and other odour domains (e.g., threshold and discrimination) has been rarely studied.
Meanwhile, olfaction was reported as an age-related function among general adults, but many studies did not include age as a confounding factor when detecting olfactory impairment among patients with schizophrenia $[11,19]$. Even though many studies about olfactory impairment among patients with schizophrenia have been conducted, few studies aimed at reporting the relation between olfaction and schizophrenia in Chinese patients. Addressing these limitations, we want to conduct a case-control study with the aim of detecting three olfactory performances in Chinese schizophrenia patients compared with healthy control subjects, and elucidating the sexual dimorphism in the relationship between psychotic symptom and olfactory function among schizophrenia patients, with the consideration of age effect, to provide useful information for further olfactory study in schizophrenia and clinical practice.

\section{Methods \\ Procedure and participates}

The aim of this study was to detect differences in responses to three olfactory performances in Chinese schizophrenia patients compared with healthy control subjects and to examine the sexual dimorphism in the relationship between psychotic symptoms and olfactory function among schizophrenia patients, with the consideration of age effect.

For this study, we recruited 110 patients aged 1835 years old who were diagnosed with schizophrenia (66 males, 44 females). The patients were diagnosed according to DSM-V; patients were excluded for neurological disorder, organic brain injury, any other mental illness, any major olfactory disturbance histories or general poor somatic health. These participants were all in the first-episode stage of schizophrenia and naive to any antipsychotic medication prior to the olfactory testing. Young adults (18-35 years old) were selected as participants for two reasons: one was that in previous studies of the "Sniffin' Sticks" test (SST) in a general population, a difference was found in olfactory performance among different adult age groups (e.g., young adult, middle-aged adult and older adult) [11, 19]; the other reason was that first-episode schizophrenia is most often seex in the young adult (18-35 years old) was the main age group.

Healthy controls, 110 community-based subjects aged 18-35 years, were recruited by advertising and were free of any psychotic disorders according to DSM-V. The exclusion criteria were in accordance with the schizophrenia group; additionally, individuals with a family history of psychosis were excluded. Age ( \pm 3 years) and sex were matched for each control. The demographic details are shown in Table 1. All healthy participants and guardians of patients provided written informed consent. 


\section{Psychotic symptom assessment}

Psychotic symptoms were assessed by two psychiatrists using the Positive and Negative Syndrome Scale (PANSS) and the interrater reliability was 0.81 . This scale included positive, negative and general psychopathy subscales [20].

\section{Olfactory testing}

The Sniffin' Sticks Test, with a high repeatability, was used to evaluate olfaction [21, 22]. It is composed of three tests-odour threshold, odour discrimination and odour identification. The odour threshold task consists of 16 types of n-butanol solution with different concentrations, which start from $4 \%$. Triplet of felt-tipped pens is a group with two containing blank solvent and a third containing the odorant of n-butanol solution, and the triplets are presented to the subject in randomized order. The subject is asked to determine the odorant from the other two blank solvent.

The odour discrimination task comprises 16 groups as well. A group, consisting of a triplet of pens, includes two with the same odorant and a third with a different odorant. A triplet of pens is randomly presented to the subject. The subject has to identify which one smelled different.

During the odour identification test, 16 odours were presented to participants through the felt-tipped pen dispensers and each odour must be identified by the subject from a list of four descriptors.

In the three sub-tests, the interval between the presentation of each pen and each group was approximately $3 \mathrm{~s}$ and 20-30 s, respectively. As the score of each sub-test ranges from 0 to 16 and the total score, which was the TDI score comprising threshold, discrimination and identification, ranges from 0 to 48 .

\section{Statistical analyses}

Continuous data were described by mean or median and compared between the schizophrenia and control groups by $t$ test, or non-parametric test if the data did not meet normal distribution. The Kolmogorov-Smirnov test was used to assess the normality. Categorical data were compared by chi-square test between the two groups. Partial correlation was used to control confounding factors and

Table 1 Demographic characteristics and olfactory performance scores

\begin{tabular}{|c|c|c|c|}
\hline & Schizophrenia & Healthy & $t$ \\
\hline & $n=110$ (male: 66;female:44) & $\mathrm{n}=110$ (male:66;female:44) & \\
\hline Age, mean (SD) in years & $22.86(4.61)$ & $23.46(4.18)$ & 5265 (Mann-Whitney) \\
\hline M & $22.85(4.69)$ & $24.47(4.80)$ & $1737^{*}$ \\
\hline $\mathrm{F}$ & $22.89(4.56)$ & $22.00(2.40)$ & 967 \\
\hline \multicolumn{4}{|l|}{ Sex, N (p) } \\
\hline M & 66 & 66 & $0\left(x^{2}\right)$ \\
\hline $\mathrm{F}$ & 44 & 44 & \\
\hline Smoking & $21(19.1)$ & $8(7.3)$ & $6.7^{*}\left(x^{2}\right)$ \\
\hline M & $19(28.79)$ & $8(12.12)$ & $5.63^{*}\left(x^{2}\right)$ \\
\hline $\mathrm{F}$ & $2(4.54)$ & 0 & $2.05\left(x^{2}\right)$ \\
\hline \multicolumn{4}{|l|}{ SST, mean (SD) in score } \\
\hline Threshold & $5.98(2.77)$ & $8.75(3.18)$ & $6.89^{* *}$ \\
\hline M & $5.92(3.08)$ & $8.92(3.28)$ & $5.42^{* *}$ \\
\hline $\mathrm{F}$ & $6.07(2.25)$ & $8.50(3.05)$ & $4.26^{* *}$ \\
\hline Discrimination & $8.73(3.55)$ & $12.44(1.79)$ & $9.80^{* *}$ \\
\hline M & $8.62(3.82)$ & $12.55(1.76)$ & $7.58^{* *}$ \\
\hline $\mathrm{F}$ & $8.89(3.13)$ & $12.27(1.84)$ & $6.20^{* *}$ \\
\hline Identification & $10.60(2.73)$ & $13.02(1.72)$ & $7.12^{* *}$ \\
\hline M & $10.09(3.00)$ & $13.14(1.76)$ & $6.99^{* *}$ \\
\hline $\mathrm{F}$ & $11.36(2.09)$ & $12.84(1.66)$ & $3.67^{* *}$ \\
\hline TDI & $25.31(6.65)$ & $34.21(4.98)$ & $11.23^{* *}$ \\
\hline M & $24.64(7.24)$ & $34.61(5.09)$ & $9.15^{* *}$ \\
\hline $\mathrm{F}$ & $26.32(5.58)$ & $33.61(4.81)$ & $6.57^{* *}$ \\
\hline
\end{tabular}

SST the Sniffin' sticks test, $M$ Male, $F$ Female, TDI the total score of threshold, discrimination and identification ${ }^{*}: P<0.05 ;{ }^{* *}: P<0.01$ 
show the relationship between clinical scales (PANSS) and the SST scores. An alpha level of below 0.05 was defined as statistical significance. The significant $P$ value was corrected by Bonferroni Correction for multiple testing. All data analyses were performed with the statistical package SPSS (version 16.0).

\section{Results}

Demographic factors, smoking status and the SST scores (including olfactory threshold, discrimination, identification and TDI score) are displayed in Table 1. The average age was $22.86(\mathrm{SD}=4.61)$ and $23.46(\mathrm{SD}=4.18)$ years for the schizophrenia and healthy group, respectively. There was no significant difference in the age and sex between the schizophrenia and healthy group. The schizophrenia participants showed lower mean scores of SST test than the control group among both males and females (Table 1 and Fig. 1). Among the schizophrenia group, the difference in smoking status shows statistical significance between male and female $\left(x^{2}=10.01, P<0.01\right)$.

The olfactory performances and psychotic symptoms for male and female schizophrenia patients are illustrated in Table 2. The positive, negative and general psychopathology subscale scores, and the five factor symptom scores did not show a statistical difference; the difference of the identification score had statistical significance $(t=-2.45, P<0.05)$, while the threshold, discrimination and TDI scores did not.

The threshold (KS $\mathrm{Z}=1.69, p<0.05$ ), discrimination (KS $\mathrm{Z}=1.85, p<0.05$ ), identification (KS $\mathrm{Z}=1.75, p<$ $0.05)$ and TDI (KS Z $=1.14, p<0.05)$ scores did not meet the normal distribution, nor did the positive symptom score (KS $\mathrm{Z}=1.38, p=0.046)$. The negative symptom score met the normal distribution (KS $\mathrm{Z}=0.94, p=$ $0.35)$. Correlation between psychotic symptoms and olfactory performance in the schizophrenia group is illustrated in Fig. 2. Controlling for the confounding factor, in male schizophrenia participants, the negative subscale score showed a significant inverse correlation with discrimination $(\gamma=-0.37, p<0.008)$, identification $(\gamma=-$ $0.45, p<0.008)$ and TDI $(\gamma=-0.50, \mathrm{p}<0.008)$ scores; the general psychopathology subscale score was inversely and significantly correlated with identification $(\gamma=-0.47, p<$ $0.008)$, and the TDI $(\gamma=-0.41, p<0.008)$ scores.

In contrast, among female schizophrenia patients, positive and general psychopathology subscale scores appeared to have a significant inverse correlation with the identification score (positive: $\gamma=-0.47, p<0.008$; general psychopathology: $\gamma=-0.42, p<0.008$ ).

\section{Discussion}

Considering the effect of age, we conducted this case-control study to explore the olfactory function of first-episode schizophrenia patients aged $18-35$ in China and the sex effect on the relationship between olfaction and schizophrenia with the consideration of the smoking confounder. In this study, olfactory impairment was found among Chinese schizophrenia patients. In terms of psychotic symptoms, negative symptoms were correlated with odour discrimination and identification in male schizophrenia patients, whereas positive symptoms were correlated with odour identification in female schizophrenia patients. General psychopathy symptom was correlated with odour identification both in male and female schizophrenia patients.

In this study, matched by sex and age, schizophrenia patients showed poorer performance across all the measures of olfaction, compared with the healthy group. Although many researchers have reported olfactory impairment in schizophrenia since 20 years ago [23], few have reported about olfactory performance among those with schizophrenia. Recently, a study conducted in China reported an odour identification deficit in patients with schizophrenia [24]; unfortunately, this study only detected the odour identification performance instead of the whole olfactory function of Chinese patients with schizophrenia.

After adjusting for the smoking status, odour identification was correlated with negative and positive symptoms among male and female schizophrenia patients, respectively. The extant literature had noted a robust correlation between odour identification and negative symptoms in schizophrenia $[25,26]$ and many studies further showed sex effect on olfactory function both in schizophrenia $[27,28]$ and in general population $[11,22]$. Further studies considering sex effect indicated a high correlation between negative symptoms and odour identification only in male schizophrenia patients [29]. Our study is strongly consistent with

Table 2 Comparison of PANSS scores and the SST scores between male and female schizophrenia

\begin{tabular}{llll}
\hline & Male & Female & $t$ \\
& $n=66$ & $n=44$ & \\
\hline $\begin{array}{l}\text { SST score, mean (SD) } \\
\text { Threshold }\end{array}$ & $5.92(3.08)$ & $6.07(2.25)$ & -.27 \\
$\quad$ Discrimination & $8.62(3.82)$ & $8.89(3.13)$ & -.38 \\
Identification & $10.09(3.00)$ & $11.36(2.09)$ & $-2.45^{*}$ \\
TDI & $24.64(7.24)$ & $26.32(5.58)$ & -1.30 \\
PANSS score, mean (SD) & & & \\
Sub-scale score & & & \\
$\quad$ Positive & $16.26(4.97)$ & $15.82(4.60)$ & .47 \\
$\quad$ Negative & $16.80(6.22)$ & $15.89(5.23)$ & .81 \\
$\quad$ General Psychopathy & $31.76(8.40)$ & $30.52(7.49)$ & .79 \\
\hline
\end{tabular}

PANSS the positive and negative syndrome scale, SST the Sniffin' sticks test, TDI the total score of threshold, discrimination and identification ${ }^{*}: P<0.05 ;{ }^{* *}: P<0.01$ 


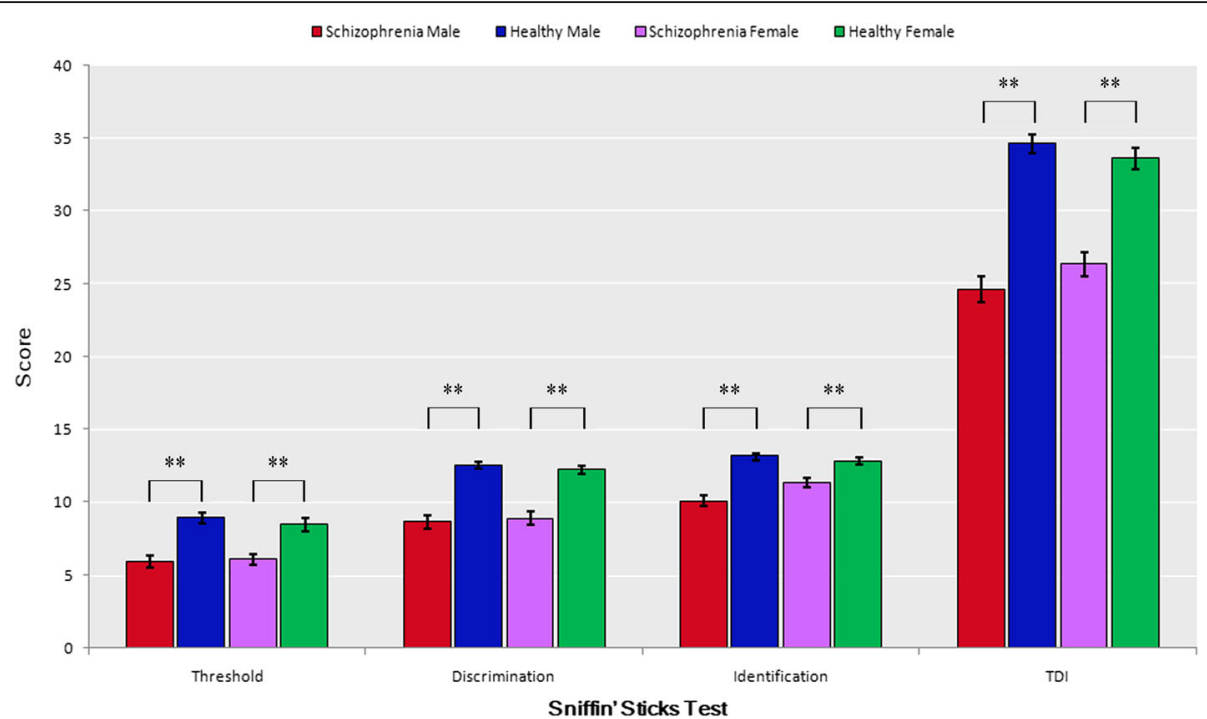

Fig. 1 Mean "Sniffin' Sticks" test scores with standard error bars by diagnosis and sex. Abbreviations TDI, the total score of threshold, discrimination and identification

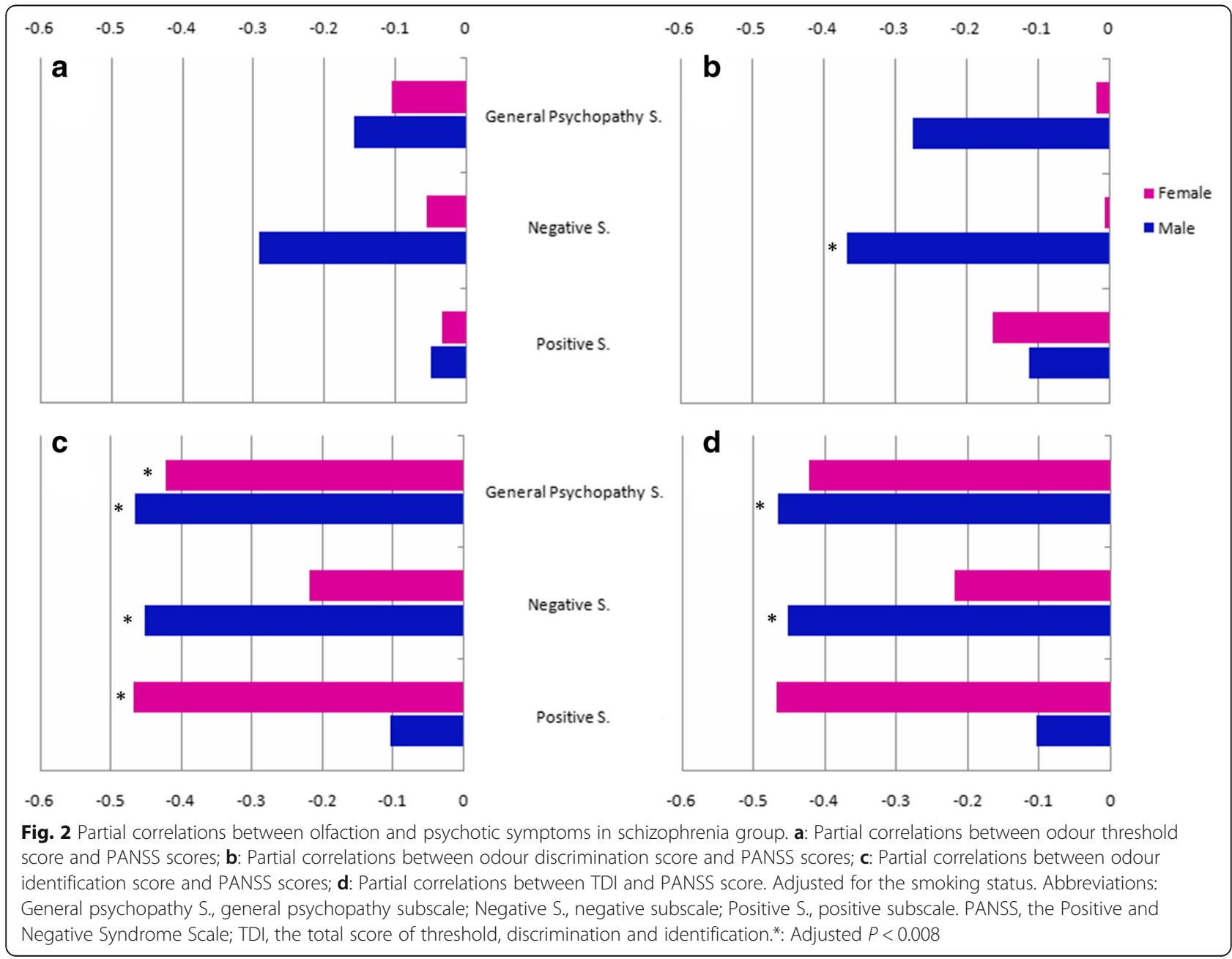


this finding that poorer odour identification was only related to negative symptoms in male schizophrenia patients. In addition, a different finding that olfactory identification was correlated with positive symptoms among female schizophrenia patients still remains in this study. This was scarcely reported before. Only a recently study found fewer serious positive symptoms were associated with better odour detection threshold for citralva [30].We think whether considering the effect of sex or not might account for the different reporting. Moreover, because the negative symptom of schizophrenia have been reported to be correlated with olfactory impairment by researchers starting two decades ago [13], many following studies have focused on exploring negative symptoms and might neglect positive symptoms when detecting olfactory function in patients with schizophrenia [12].

Meanwhile, sex dimorphism exists in the symptomatology, antipsychotic response [28], social functioning [14], illness characteristics [15] and cerebral structure [16] of schizophrenia. It might be helpful to consider these factors, as well as hormone effect [28], when future studies try to elucidate the sex effect on olfaction. Meanwhile, odour identification has been described as a central process [31] and the limbic structure (e.g., amygdala and the orbitofrontal cortex) was the location of olfactory association cortex. The abnormality of limbic structure was not only connected with olfactory dysfunction [31] but also connected with negative symptomatology [32]. Furthermore, the orbitofrontal cortex to amygdala ratio presented sex dimorphism [33], and hyperfrontality and hypofrontality was correlated with positive and negative symptoms, respectively [34, 35]. Collectively, these might provide another possible explanation for the sex difference presented regarding the relationship between olfactory impairment and symptomatology. Moreover, the sex related difference of olfactory bulb [36] might also be a possible way to elucidate this result. In addition, Chinese patients were participants in this study, for whom the sex effect on olfaction was hardly reported. It might be possible that there is heterogeneity of olfactory impairment in Chinese schizophrenia patients.

Odour discrimination, studied much less than olfactory identification in previous research [8], was related to negative symptoms among male patients in this study. Odour discrimination being more central processing when compared with odour threshold, and its deficit is related to the hippocampus and amygdala, which are also the impaired brain region among schizophrenia [31]. Therefore, the relation between odour discrimination and negative symptoms in male schizophrenia patients should be detailed further. Expectedly, general psychopathy, including cognition, was correlated with odour identification. This was in consistent with previous studies that have found that olfactory identification was strongly related with cognition and it was considered to be a useful supplementary screening tool of Alzheimer's disease [37].

The limitation of this study was the lack of neuroimaging or electrophysiological studies to further explain why different sex shows different relationships between psychotic symptoms and olfactory impairment. This is the direction of our further studies.

\section{Conclusion}

Sex influenced the relationship between psychotic symptoms and olfactory impairment in first-episode schizophrenia patients aged 18-35 years. Negative and positive symptoms were associated with odour identification deficit in male and female schizophrenia, respectively. The odour discrimination deficit was correlated with negative symptom among male schizophrenia patients. The effect of sex on olfactory impairment among patients with schizophrenia should be studied more profoundly.

\section{Abbreviations}

PANSS: Positive and Negative Syndrome Scale; SST: The Sniffin' Sticks Test; TDI: The total score of threshold, discrimination and identification

\section{Acknowledgements}

We thank Professor Xiehe Liu, who kindly provided guidance on this research.

\section{Funding \\ The design of the study, and collection, analysis and interpretation of data are supported by the National Natural Science Foundation of China (No. 81401132). The publication of the paper was supported by the Key Research and Development Project of Province Science and Technology Department, Sichuan (No. 2017SZ0062).}

Availability of data and materials

The datasets used and/or analysed during the current study are available from the corresponding author on reasonable request.

\section{Authors' contributions}

XC and JX contributed equally. Study concept and design: JX, XC and JH. Data collection: JX, XC. Analysis and interpretation of data: XC, JX. Drafting of the manuscript: $X C$ and JX. Critical revision of the manuscript for important intellectual content: JH, WG, BL, JZ. All authors reviewed and approved the manuscript.

\section{Ethics approval and consent to participate}

This study was approved by the Ethical Review Board of the West China Hospital, Sichuan University. The approval from officials of sampled hospital was obtained in a written form. Legal guardians were contacted to provide written consent on behalf of the patients to participate in the study.

\section{Consent for publication}

Not applicable.

\section{Competing interests}

The authors declare that they have no competing interests.

\section{Publisher's Note}

Springer Nature remains neutral with regard to jurisdictional claims in published maps and institutional affiliations. 
Received: 31 December 2017 Accepted: 12 June 2018

Published online: 18 June 2018

\section{References}

1. Kahn RS, Keefe RSE. Schizophrenia is a cognitive illness: time for a change in focus. Jama Psychiatry. 2013;70(10):1107.

2. Turetsky BI, Hahn CG, Borgmann-Winter K, Moberg PJ. Scents and nonsense: olfactory dysfunction in schizophrenia. Schizophr Bull. 2009;35(6):1117-31.

3. Nolte J, S J. The human brain: an introduction to its functional anatomy. 6 th ed. Philadelphia: Mosby; 2008.

4. Ugur T, Weisbrod M, Franzek E, Pfuller U, Sauer H. Olfactory impairment in monozygotic twins discordant for schizophrenia. Eur Arch Psychiatry Clin Neurosci. 2005:255(2):94-8

5. Ishizuka K, Tajinda K, Colantuoni C, Morita M, Winicki J, Le C, Lin S, Schretlen D, Sawa A, Cascella NG. Negative symptoms of schizophrenia correlate with impairment on the University of Pennsylvania smell identification test. Neurosci Res. 2010;66(1):106-10.

6. Amsterdam JD, Settle RG, Doty RL, Abelman E, Winokur A. Taste and smell perception in depression. Biol Psychiatry. 1987;22(12):1481-5.

7. Rupp Cl, Fleischhacker WW, Kemmler G, Oberbauer H, Scholtz AW, Wanko C, Hinterhuber $\mathrm{H}$. Various bilateral olfactory deficits in male patients with schizophrenia. Schizophr Bull. 2005;31(1):155-65.

8. Nguyen AD, Shenton ME, Levitt JJ. Olfactory dysfunction in schizophrenia: a review of neuroanatomy and psychophysiological measurements. Harv Rev Psychiatry. 2010;18(5):279-92.

9. Pelka-Wysiecka J, Wronski M, Bienkowski P, Murawiec S, Samochowiec A Samochowiec J. Odors identification differences in deficit and nondeficit schizophrenia. Pharmacol Rep. 2016;68(2):390-5.

10. Cohen AS, Brown LA, Auster TL. Olfaction, "olfiction", and the schizophreniaspectrum: an updated meta-analysis on identification and acuity. Schizophr Res. 2012;135(1-3):152-7.

11. Hummel T, Kobal G, Gudziol H, Mackaysim A. Normative data for the "Sniffin' sticks" including tests of odor identification, odor discrimination, and olfactory thresholds: an upgrade based on a group of more than 3,000 subjects. Eur Arch Otorhinolaryngol. 2007;264(3):237-43.

12. Malaspina D, Goetz R, Keller A, Messinger JW, Bruder G, Goetz D, Opler M, Harlap S, Harkavy-Friedman J, Antonius D. Olfactory processing, sex effects and heterogeneity in schizophrenia. Schizophr Res. 2012;135(1-3):144-51.

13. Moberg PJ, Agrin R, Gur RE, Gur RC, Turetsky BI, Doty RL. Olfactory dysfunction in schizophrenia : a qualitative and quantitative review. Neuropsychopharmacol Official Publ Am Coll europsychopharmacol. 1999; 21(3):325-40.

14. Andia AM, Zisook S, Heaton RK, Hesselink J, Jernigan T, Kuck J, Morganville J, Braff DL. Gender differences in schizophrenia. J Nerv Ment Dis. 1995; 183(8):522-8.

15. Ochoa S, Usall J, Cobo J, Labad X, Kulkarni J. Gender differences in schizophrenia and first-episode psychosis: a comprehensive literature review. Schizophrenia Res Treat. 2012;2012:916198.

16. Crow TJ, Chance SA, Priddle TH, Radua J, James AC. Laterality interacts with sex across the schizophrenia/bipolarity continuum: an interpretation of meta-analyses of structural MRI. Psychiatry Res. 2013;210(3):1232-44.

17. Beery AK, Zucker I. Sex bias in neuroscience and biomedical research. Neurosci Biobehav Rev. 2011;35(3):565-72

18. Good KP, Leslie RA, McGlone J, Milliken HI, Kopala LC. Sex differences in olfactory function in young patients with psychotic disorders. Schizophr Res. 2007;97(1-3):97-102

19. Sorokowska A, Schriever VA, Gudziol V, Hummel C, Hähner A, lannilli E, Sinding C, Aziz M, Seo HS, Negoias S. Changes of olfactory abilities in relation to age: odor identification in more than 1400 people aged 4 to 80 years. Eur Arch Otorhinolaryngol. 2015;272(8):1937-44.

20. Kay SR, Opler LA, Lindenmayer JP. Reliability and validity of the positive and negative syndrome scale for schizophrenics. Psychiatry Res. 1988;23(1):99-110.

21. Kobal G, Klimek L, Wolfensberger M, Gudziol H, Temmel A, Owen CM, Seeber H, Pauli E, Hummel T. Multicenter investigation of 1,036 subjects using a standardized method for the assessment of olfactory function combining tests of odor identification, odor discrimination, and olfactory thresholds. Eur Arch Otorhinolaryngol. 2000;257(4):205-11.

22. Kobal G, Hummel T, Sekinger B, Barz S, Roscher S, Wolf S. "Sniffin' sticks": screening of olfactory performance. Rhinology. 1996;34(4):222-6.

23. Hurwitz T, Kopala L, Clark C, Jones B. Olfactory deficits in schizophrenia. Biol Psychiatry. 1988;23(2):123-8.
24. Zou LQ, Zhou HY, Lui SSY, Wang Y, Wang Y, Gan J, Zhu XZ, Cheung EFC, Chan RCK. Olfactory identification deficit and its relationship with hedonic traits in patients with first-episode schizophrenia and individuals with schizotypy. Prog Neuro-Psychopharmacol Biol Psychiatry. 2018:83:137-41.

25. Good KP, Whitehorn D, Rui Q, Milliken H, Kopala LC. Olfactory identification deficits in first-episode psychosis may predict patients at risk for persistent negative and disorganized or cognitive symptoms. Am J Psychiatr. 2006; 163(163):932-3.

26. Good KP, Tibbo P, Milliken H, Whitehorn D, Alexiadis M, Robertson N, Kopala LC. An investigation of a possible relationship between olfactory identification deficits at first episode and four-year outcomes in patients with psychosis. Schizophr Res. 2010;124(1-3):60-5.

27. Highley JR, DeLisi LE, Roberts N, Webb JA, Relja M, Razi K, Crow TJ. Sexdependent effects of schizophrenia: an MRI study of gyral folding, and cortical and white matter volume. Psychiatry Res. 2003;124(1):11-23.

28. Abel KM, Drake R, Goldstein JM. Sex differences in schizophrenia. Int Rev Psychiatry. 2010;22(5):417-28.

29. Urban M, Rabe-Jablonska J. Olfactory dysfunctions in patients with schizophrenia. Psychiatr Pol. 2007:41(4):503-12.

30. Kamath $V$, Lasutschinkow $P$, Ishizuka K, Sawa A. Olfactory functioning in firstepisode psychosis. Schizophr Bull. 2017:44(3):672-80.

31. Rupp Cl, Fleischhacker WW, Kemmler G, Kremser C, Bilder RM, Mechtcheriakov S, Szeszko PR, Walch T, Scholtz AW, Klimbacher M, et al. Olfactory functions and volumetric measures of orbitofrontal and limbic regions in schizophrenia. Schizophr Res. 2005;74(2-3):149-61.

32. Rahm C, Liberg B, Reckless G, Ousdal O, Melle I, Andreassen OA, Agartz I. Negative symptoms in schizophrenia show association with amygdala volumes and neural activation during affective processing. Acta Neuropsychiatrica. 2015;27(4):213-20.

33. Gur RE, Kohler C, Turetsky BI, Siegel SJ, Kanes SJ, Bilker WB, Brennan AR, Gur RC. A sexually dimorphic ratio of orbitofrontal to amygdala volume is altered in schizophrenia. Biol Psychiatry. 2004;55(5):512-7.

34. Semkovska M, Bedard MA, Stip E. Hypofrontality and negative symptoms in schizophrenia: synthesis of anatomic and neuropsychological knowledge and ecological perspectives. Encephale. 2001;27(5):405-15.

35. Shinto AS, Kamaleshwaran KK, Srinivasan D, Paranthaman S, Selvaraj K, Pranesh MB, Lakshminarayanan GN, Prakash B. "Hyperfrontality" as seen on FDG PET in unmedicated schizophrenia patients with positive symptoms. Clin Nucl Med. 2014;39(8):694-7.

36. Oliveira-Pinto AV, Santos RM, Coutinho RA, Oliveira LM, Santos GB, Alho AT, Leite RE, Farfel JM, Suemoto CK, Grinberg LT, et al. Sexual dimorphism in the human olfactory bulb: females have more neurons and glial cells than males. PLoS One. 2014;9(11):e111733.

37. Quarmley M, Moberg PJ, Mechanic-Hamilton D, Kabadi S, Arnold SE, Wolk DA, Roalf DR. Odor identification screening improves diagnostic classification in incipient Alzheimer's disease. J Alzheimers Dis Jad. 2016;55(4):1497-507.

\section{Ready to submit your research? Choose BMC and benefit from:}

- fast, convenient online submission

- thorough peer review by experienced researchers in your field

- rapid publication on acceptance

- support for research data, including large and complex data types

- gold Open Access which fosters wider collaboration and increased citations

- maximum visibility for your research: over $100 \mathrm{M}$ website views per year

At BMC, research is always in progress.

Learn more biomedcentral.com/submissions 